\title{
Coups de sonde génétiques dans « Les mers du sud »
}

\section{Maura Bonfiglio}

\section{(2) OpenEdition}

1 Journals

Édition électronique

URL : https://journals.openedition.org/genesis/1791

DOI : 10.4000/genesis. 1791

ISSN : 2268-1590

Éditeur :

Presses universitaires de Paris Sorbonne (PUPS), Société internationale de génétique artistique littéraire et scientifique (SIGALES)

Édition imprimée

Date de publication : 9 mai 2017

Pagination : 183-195

ISBN : 979-1023-105636

ISSN : 1167-5101

\section{Référence électronique}

Maura Bonfiglio, "Coups de sonde génétiques dans « Les mers du sud » », Genesis [En ligne], 44 |

2017, mis en ligne le 22 mai 2018, consulté le 17 mars 2023. URL : http://journals.openedition.org/ genesis/1791; DOI : https://doi.org/10.4000/genesis.1791 


\title{
Coups de sonde génétiques dans «Les mers du sud»
}

\author{
Maura Bonfiglio
}

L es mers du sud» («I mari del sud»; à lire en annexe), l'un des poèmes les plus beaux et les plus connus de Cesare Pavese, ouvre Lavorare stanca (Travailler fatigue), son premier recueil poétique, dans chacune des deux éditions relues par l'auteur (Solaria 1936, Einaudi 1943). Écrit en 1930 et dédié à Augusto Monti, son professeur et guide intellectuel et politique, ce poème marque le passage de la production de jeunesse vers la phase de maturité de Pavese ${ }^{1}$. Il est centré sur un fait autobiographique : le lien affectif entre l'auteur et son cousin, dans un dialogue dépouillé, fait de mots entourés de silences et de souvenirs, à l'occasion d'une promenade à flanc de colline. La thématique dominante - chargée d'un fort investissement autobiographique - est celle de l'identité, une identité enfouie profondément, liée à la terre, à la région des Langhe, d'une force qui ne peut être éraflée même par vingt ans de pérégrinations autour du monde. La nature de cette force mystérieuse et secrète fascine Pavese et restera, tout comme le sens insaisissable de l'existence, l'objet de son incessante recherche poétique. L'importante fonction augurale du texte est liée non seulement au processus de formation de sa propre identité et à certains mythes chers à l'auteur, mais aussi à la thématique, ici en toile de fond, qui traverse tout le recueil : le paradoxe du travail, qui d'un côté est nécessaire à la subsistance mais qui conduit de l'autre à la perte de l'énergie, du temps, et donc de la vie elle-même; le travail comme image de la mort.

\section{Matériau de travail et lignes de force}

Parmi les documents qui attestent les phases d'élaboration du poème ${ }^{2}$, le premier d'un point de vue chronologique est le feuillet $51 \mathrm{r}^{\circ}$ de AP X.52 (que l'on peut dater d'août 1930), un plan thématico-structurel en prose, fondamental car d'un côté il guide une part de la rédaction et de a) Salire in silenzio a vedere il faro (Siamo quasi in cima)

$\beta)$ col cugino così e così (ghiaiaccia, automobili, soldi) («mi imbarcherei domani »)

$\gamma$ ) (Mai parlare) È stato lì e là. Ha veduto inseguire balene/tra schiume di sangue.

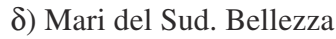

$\varepsilon)$ Langhe di notte (spirito)

$\zeta$ ) Domanda («Tuo padre amava leggere. Far soldi in famiglia. Pur non spenderli in medicine)

$\eta)$ il faro della città e del mondo sognati da bambini

$\theta)$ È morto. Li ha spesi in medicine. La vita è dura. E smania 3 .

Fig. 1 : Transcription du feuillet AP X.52, $51 \mathrm{r}^{\circ}$

1.Les débuts littéraires de Pavese ont été publiés seulement après sa mort. Un recueil de poèmes initié en 1923 (Rose gialle in una coppa nera) deviendra Sfoghi (1923-1926); Pavese compose entre 1925 et 1927 les poèmes de Rinascita, et entre 1928 et 1929 ceux de Blues della grande città. Il ne reste que des ébauches retrouvées dans des carnets d'un roman entamé dans les années 1924-1926 puis abandonné (Romanzo della gioventù). Il participe en 1928 au mouvement «Strabarriera», lancé par d'anciens camarades du lycée Massimo D'Azeglio, élèves du professeur Monti (la «Confraternité de D'Azeglio»), qui oppose à la ville - moderne, frénétique, chaos de lumière et de bruits - la banlieue comme lieu des marges, du désespoir et de l'inspiration des jeunes artistes. Mais la perception de la ville dans l'œuvre de Pavese est également influencée par sa lecture des Américains; c'est dans ces années qu'il travaille à sa thèse de doctorat sur Leaves of Grass ( Sur l'interprétation de la poésie de Walt Whitman », 1930).

2. L'archive de Lavorare stanca est rassemblée dans deux fonds, le Fondo Sini (AP), propriété de la famille du poète, et le Fondo Einaudi (FE), propriété de sa maison d'édition, donné à l'Archivio Cesare Pavese de l'université de Turin; ils sont consultables en ligne après requête et identification sur le portail «HyperPavese» (<www.hyperpavese.it $>$ ). J'ai proposé une description des deux fonds, ainsi qu'une transcription diplomatique des manuscrits, dans ma thèse de doctorat.

3. Transcription de Lorenzo Mondo pour son article «Fra Gozzano e Whitman : le origini di Pavese», dans Sigma, n 1, 3-4, 1964, p. 3-21. 
l'autre il sera modifié et profondément remanié (fig. 1). Suivent les cinq feuillets recto verso du premier brouillon, AP III.1 (7-14 septembre 1930), riche en interventions, qui atteste ce qui selon toute probabilité a été le premier état rédigé du poème et constitue un ajout considérable au plan 4 .

Également importants, les feuillets autographes de FE 5II.38, qui même s'ils constituent une mise au net de AP III.1, portent encore des modifications substantielles 5 . Les dactylogrammes (FE 5II.16 et FE 5II.51) et les épreuves d'imprimerie (AP III.3) montrent par contre peu de repentirs significatifs, tout au plus des variantes de ponctuation ou quelques corrections de fautes de frappe.

L'analyse génétique permet de saisir un riche processus d'élaboration textuelle, qui se déploie dans deux directions. D'un côté, le poème s'en tient au plan, développant quelques éléments à l'intérieur d'une forme qui ne bougera plus; de l'autre il s'en détache progressivement (après son amplification), en abandonnant quelques éléments clés et surtout en faisant descendre tout au bas du poème «Mari del Sud. Bellezza», qui sera développé dans la conclusion de la composition et lui donnera son titre.

Je rendrai compte de ce double mouvement à travers des échantillons qui montrent comment le premier schéma en prose accompagne la rédaction des premières strophes puis comment le premier manuscrit modifie la donne puisqu'il comporte un ajout conséquent (AP III.1.3 $\mathrm{v}^{\circ}$ et AP III.1.2 $\left.\mathrm{r}^{\circ}\right)^{6}$, que Pavese appelle intermezzo (fig. 2 et 3 ), qui provoque un brusque réexamen du projet initial et inaugure l'étape de sa réorganisation.

Ces considérations de macrostructure permettent de dégager deux tendances principales dans la genèse du poème, d'une part le développement, d'autre part le renoncement, tendances auxquelles il faut adjoindre une troisième : la tentation de ne plus apporter aucune modification. $\underline{\text { Imari del Sud }}$

Silenziesi. to ritrovo i ricerdi di un tempe

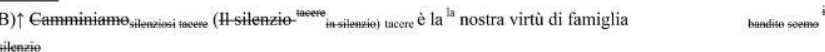

taciturno e uno scemo) qualche nostro antenato deve essere ben triste solo state $_{\text {morlo }}$ presto ${ }^{\text {stato }}$ th bandite

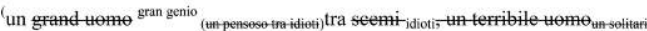

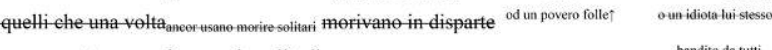
merto ceme si usava, in solitudine $\theta$ forse era seltante in centadine.

S se ha ${ }^{\text {per }}$ tramandate ${ }^{\text {insegnare }}$ ai stei ${ }^{\text {suoi }}$ tanto silenzio

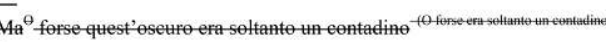
iconoseola strada degli anni perdut

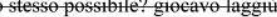

che si muove legerese simteve erangetitle pacato solermai* abbronzato nel volto Questa

sefa mi ha dette Mi ha incontrate stasera "Saliano a Mencueee?

êtrima più alta di tutte le Langhe.

fa preste. È la cima più alta-vetta) di tutte le Langhe"

Sondure passi Nelle sere Questa Stascra

Questa sera è seretroe si vede il riflesso del fare

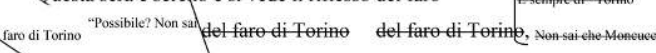

he Moncuroce è la cima piú alta di tutte lef Langhe?

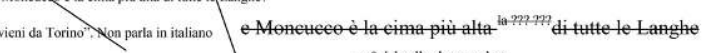
ta eima pititata di tute
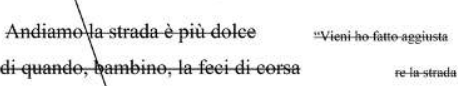

Mi dice mio qugine " $L$ 'ho fatta

Viene "Andiame, dice attera, ${ }^{\text {the }}$, ho fatto spianare la strade

Tu l'hai fatta piane da piceorema ora- ${ }^{\text {madeste }}$ ei puei essere* in

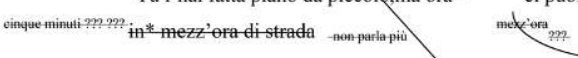

4. Légende $: \mathrm{AP}=$ Fondo Sini $;$ chiffre romain $=$ chemise $; 1^{\circ}$ numéro arabe $=$ fascicule, $2^{\circ}$ numéro arabe $=$ feuillet $; \mathrm{r}^{\circ}$ et $\mathrm{v}^{\circ}=$ recto et verso. Ce fascicule est constitué de huit pages de bifeuillets format $\mathrm{A} 4$, écrites recto verso : les cinq premières témoignent de la première rédaction du poème, la sixième et la septième contiennent l'ébauche d'un essai sur Walt Whitman, et la huitième - uniquement rédigée côté recto - est un brouillon de lettre daté du 15 septembre 1946. Le papier, ligné, est jauni, parfois froissé ou déchiré, surtout contre les marges; l'encre utilisée est noire, même pour les nombreuses et substantielles révisions. Les brouillons sont estampillés par l'institution de conservation, sur chaque recto, dans l'angle inférieur gauche du feuillet.

5. Légende $: \mathrm{FE}=$ Fonds Einaudi $; 5 \mathrm{II}=$ chemise $; 38=$ fascicule. $\mathrm{Ce}$ fascicule est composé d'un bifeuillet de format «protocole», dont les pages sont écrites recto et verso, à l'encre noire. Certaines ratures sont portées au crayon, comme certaines reformulations et insertions. Le papier à carreaux est jauni et déchiré aux endroits où il était plié en croix. 6. Ce qui est chronologiquement la deuxième page de AP III.1 a été, sans doute par erreur, classé comme AP III.1.3. De même, la troisième page de cet ensemble a été classée par erreur comme AP III.1.2. Les deux pages ont donc été interverties par les archivistes. Dans ce travail, nous respecterons le premier classement, mais l'analyse et le commentaire suivront évidemment l'ordre de l'écriture. 


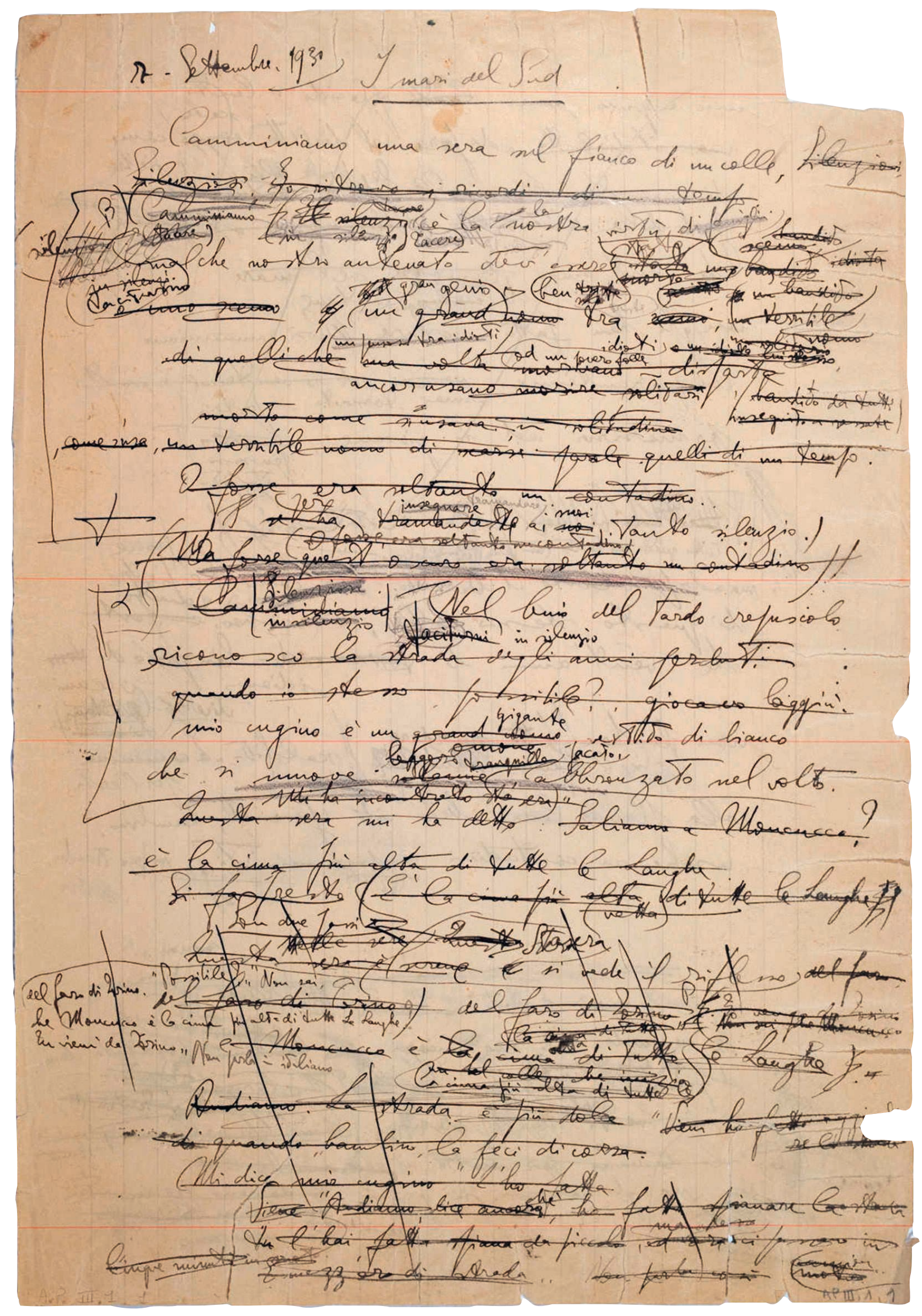

Fig. 2 : Feuillet AP III.1.1 $\mathrm{r}^{\circ}$ (Guido Gozzano - Cesare Pavese, univ. di Torino, www.hyperpavese.it) 


\section{«Monter en silence» : une ouverture très travaillée}

Pavese développe sans attendre, sur la première page du premier brouillon (AP III.1.1 $\mathrm{r}^{\circ}$, daté du «7 settembre $1931 »$, fig. 2), les points $\alpha, \beta, \eta$ du plan.

La première partie du point $\beta$ est développée dans ce qui apparaît déjà comme le début de la première strophe (délimitée par une accolade et précédée de la lettre $\mathrm{A}$ ). Le point $\eta^{7}$ trouve également sa réalisation dans la première strophe, qui reprend la référence au phare. Quant au point $\alpha$, il est surtout repris dans le vers initial de AP III.1.1 $\mathrm{r}^{\circ}$, «Camminiamo una sera sul fianco di un colle» (Un soir nous marchons le long d'une colline), qui insiste sur la marche à deux, en atténuant la verticalité de «salire» et en accentuant la progression du mouvement, avec un rythme qui semble mimer l'avancée des protagonistes. Il est d'ailleurs significatif que sur un feuillet travaillé par de multiples modifications, ce vers apparaisse déjà sous sa forme achevée, mais ce n'est pas du tout un «don de Dieu»; il semble plutôt que Pavese ait profondément réélaboré les motifs présents dans l'incipit d'un poème qu'il venait d'achever (en août 1930), «Frasi all'innamorata» (Phrases pour l'amoureuse) : «Passeggiamo in silenzio con una bambinalabbordata per strada, lungo il viale, di sera ${ }^{8}$ " (Nous marchons en silence moi et une enfant/rencontrée dans la rue, le long du boulevard, un soir). «Nous marchons» est ici un succédané du dialogue, il traduit par une image concrète le rapport au cousin; la référence temporelle («un soir») transforme l'atmosphère vespérale en moment propice à la rencontre de l'autre, et suspend l'épisode dans une «éternité sacrée ${ }^{9}$ » comme celle du souvenir, qui pourtant, réactualisé par le présent («nous marchons»), touche par son évidence; «à flanc de colline» renvoie à un dense réseau de références littéraires 10 .
L'hésitation sur la manière de qualifier l'attitude taciturne des deux marcheurs dans ce premier état rédigé du texte est également intéressante à relever. Pavese alterne plusieurs fois «silenziosi», «taciturni » et «in silenzio», mais en fin de compte revient à la forme adverbiale du plan. Ainsi, «in silenzio», qui dans «Frasi all'innamorata» était un indice de la «sazietà del terzo appuntamento» (la satiété du troisième rendez-vous) d'une relation occasionnelle,

7. Qu'il s'agisse de la Mole Antonelliana ou du Sanctuaire de la Madonna delle Grazie sur la colline de Superga, le terme fait de la ville, en langage nautique, le «point notable» de la «navigation» existentielle du poète. 8. Lorenzo Mondo le signalait dans son article «Fra Gozzano e Whitman : le origini di Pavese», Sigma, n 1, 3-4, 1964, p. 3-21.

9. Cesare Pavese, «Feria d'agosto», dans Opere di Cesare Pavese, 5, Einaudi, Torino, 1968, p. 140 (sacrale eternità).

10. Surtout à Pétrarque évoquant son ascension du mont Ventoux (Familiares, IV 1). 


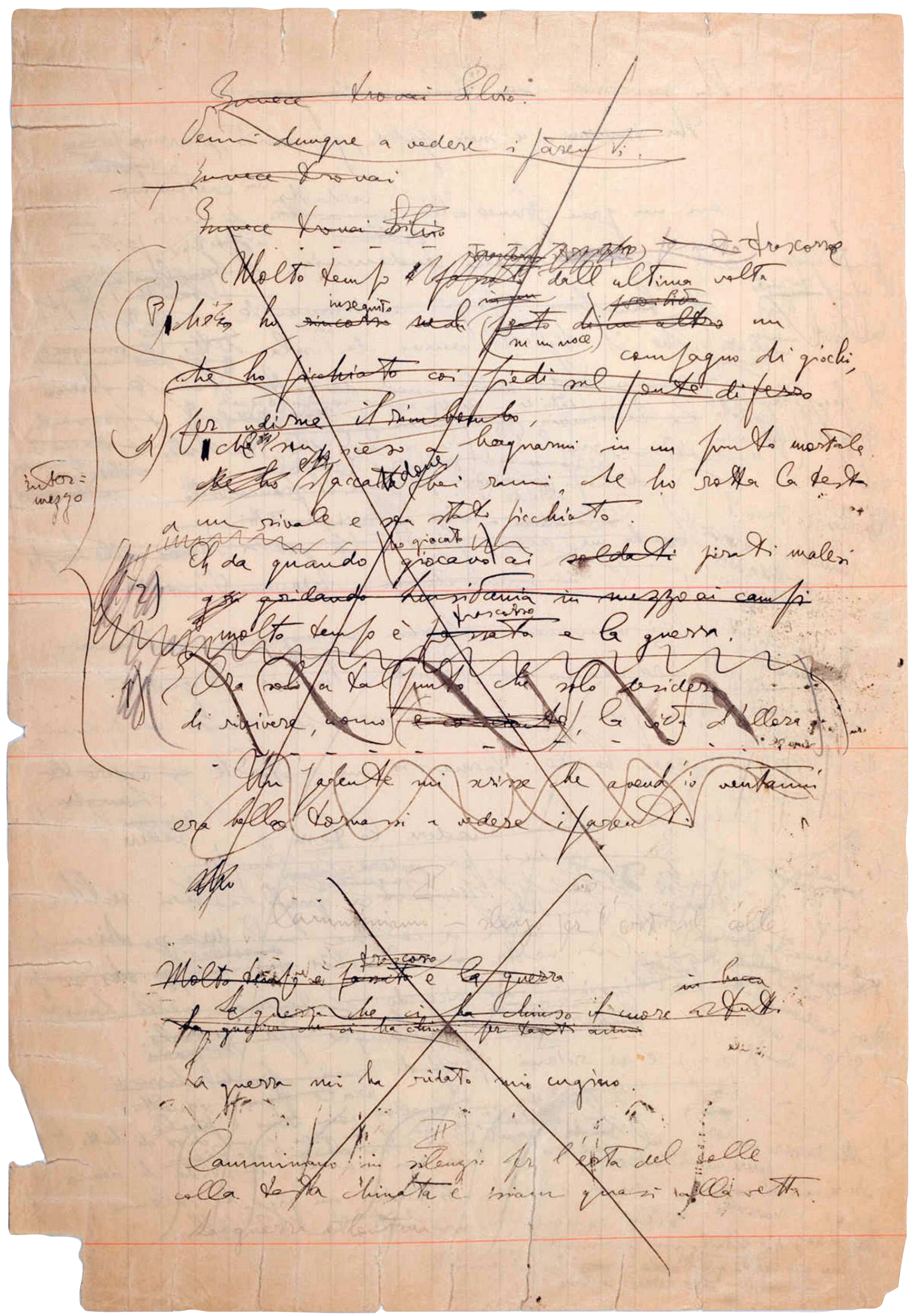

Fig. 3 : Fº AP III.1.3 v (Guido Gozzano - Cesare Pavese, univ. di Torino, www.hyperpavese.it) 
devient ici caractéristique «ontologique», marqueur de l'identité des deux personnages.

\section{Intermezzo : à reculons dans le temps}

Une double résurgence du passé, non thématisée dans le plan, enrichit dès le premier brouillon le développement du poème (strophes 3 et 4 du poème publié), finissant par faire sortir de ses gonds la structure prévue.

Pavese s'essaie d'abord (AP III.1.1 v ${ }^{\circ}$ et AP III.1.3 $\mathrm{r}^{\circ}$, non transcrit ici) à l'élaboration progressive du mythe du cousin, dont il a entendu parler, enfant, par les femmes du village «come in favola» (sur un ton de légende), alors que les hommes «che lo davano per morto (« qui le donnaient pour mort») sont démentis par l'arrivée d'une carte de vœux de Tasmanie, preuve tangible de ses aventures exotiques et qu'il est bien vivant.

Dans le sillage de cette rétrospective, il décide ensuite d'en introduire une autre concernant sa propre enfance, particulièrement son rapport à la terre d'origine, la région des Langhe; pensée initialement comme une brève parenthèse (un intermezzo), cette rétrospective sera ensuite amplifiée par une autre portion dédiée à son adolescence, particulièrement son rapport à la ville comme lieu de formation. Cette analepse consacrée à sa jeunesse finira ainsi par occuper toute la quatrième strophe du poème.

Dans la première élaboration du motif de l'enfance (AP III.1.3 vo , fig. 3), la distance temporelle est marquée, et en même temps Pavese exalte la présence vive du souvenir de ces expériences fondatrices, où se mêlent la fascination pour l'aventure, l'immersion dans la nature, la violence quasi animale. Ce souvenir suscite l'image d'une lointaine saison «dionysiaque», chargée d'énergie primordiale, et la perspective en est encore élargie par l'énumération
Oh da quando ${ }^{\text {ho }}$ giocave ${ }^{\text {to }}$ ai pirati malesi

quanto tempo è trascorso! e dall'ultima volta

che son sceso a bagnarmi in un punto mortale

ehe ehe $^{\mathrm{e}}$ ho inseguito/su un albero mentee un compagno di giochi

spaccandone i bei rami e ho rotto la testa

a un rivale e son stato picchiato,

quante ${ }^{a}$ vita tempe Ferine è traseersa! La città dawiver Terine lacittà

quanta vita è trascorsa! Altri giorni, altri amici giochi

altri squassi $\mathrm{di}^{\mathrm{del}}$ sangue dinnanzi a rivali

più elusivi: ${ }^{\text {i }}$ pensieri ed i sogni ${ }^{\text {pensierr" ed amori figure parole speranze }}$

ta città abbagliante, sfaceiata* di vita

Nen piùtregua

Oh quanto fui piechiato per Torine!

Fui piechiato Fui piechiato da sempliei sguardi

Furiechiate schiaciato $_{\text {da sguardi di donne }}$

da un celere da un geste, dal mette di un ueme

dal streno di ${ }^{\text {da }}$ una voce, da una strada da un libro, da un viale

Ogni cosa servi per provarmit. Ed al fondo di

sene-stato il piti abbietto di tutta una folta,

Per i sogni ho persine talvoltat Ehe seffertela fame ${ }^{\text {pena }}$

${ }^{\text {volto }}$ E ho toecato 1, estreme gli -ogni cosa iniziatat ${ }^{\text {tegegera }}$ piu triste

$\rightarrow$ I'ho-esageratat ${ }^{\text {esasperata }}$ fino ad aver da ridere

triste più solo di me stesso piangendol fino a ${ }^{1}$ queste ${ }^{\text {fondo }}$.

E talvolta ${ }^{\text {ho }}$ sognato dei campi deserti

in cimalle colline, delle strade

lente nella xallata, delle pietre

sgretolate dal sole cadencée des entreprises adolescentes. Dans ce premier jet, l'intermezzo se conclut avec l'allusion à la guerre («molto tempo è passato < trascorso > e la guerra») comme élément ultérieur qui approfondit la différence temporelle et spirituelle qui sépare de l'enfance, âge de la sécurité et de la vitalité, à laquelle s'oppose l'actuelle exténuation. On relève, juste après, plusieurs tentatives de réinstaller le discours dans le présent narratif, dans la promenade à flanc de colline, ou d'introduire la thématique du retour du cousin, si longtemps embarqué par des campagnes de pêche «dans des océans lointains ».

11. Voir n. 5. 
Ol de quand, ho inicasto ai fire ts nualen The bou xeso a haguami in $\mathrm{m}$ fun to mastac

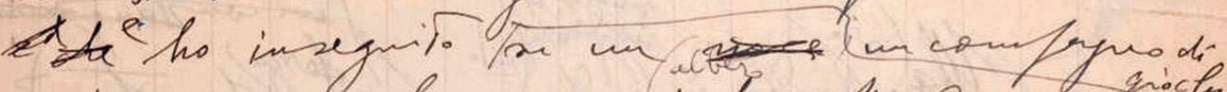

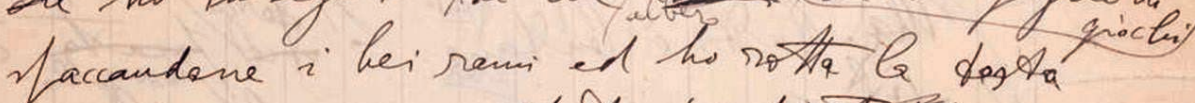
a m rivale e son stats fichioxt:

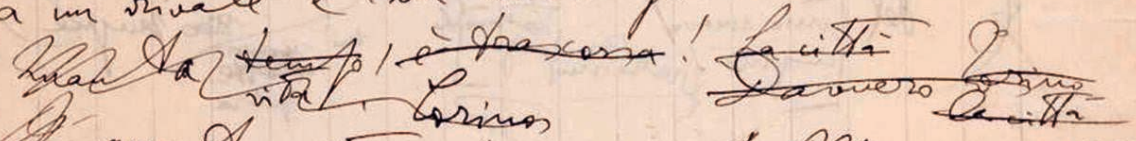

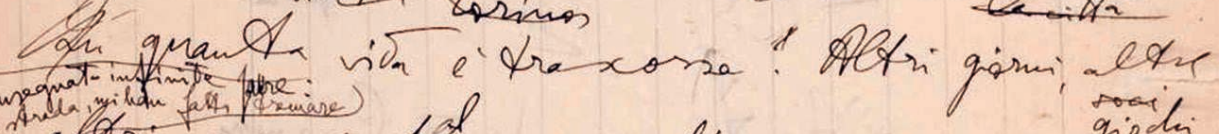
Lu

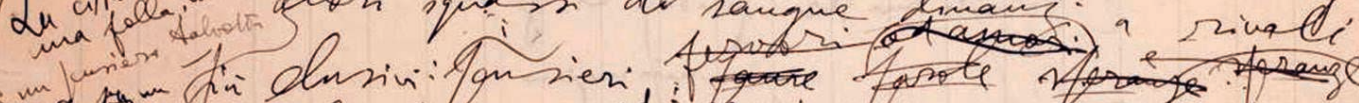

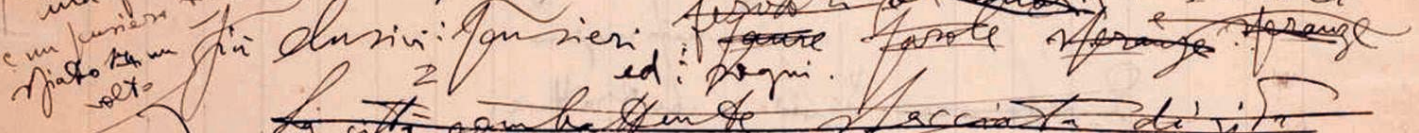

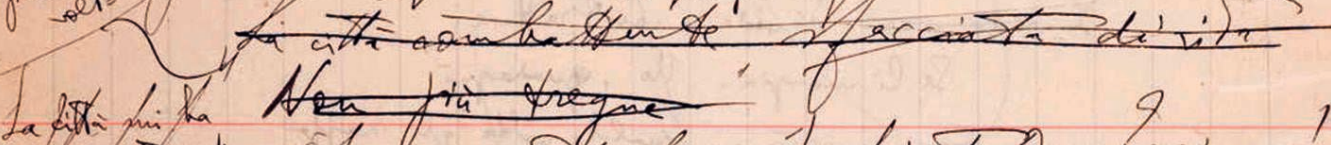

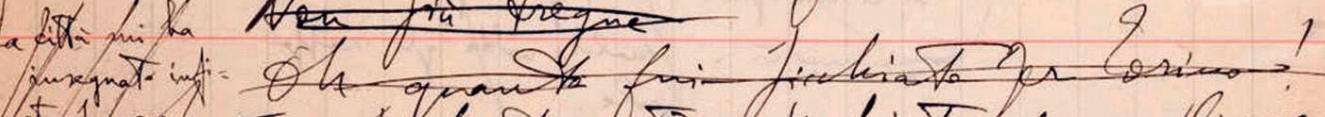

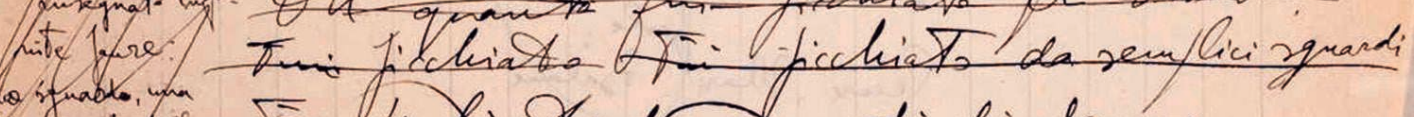

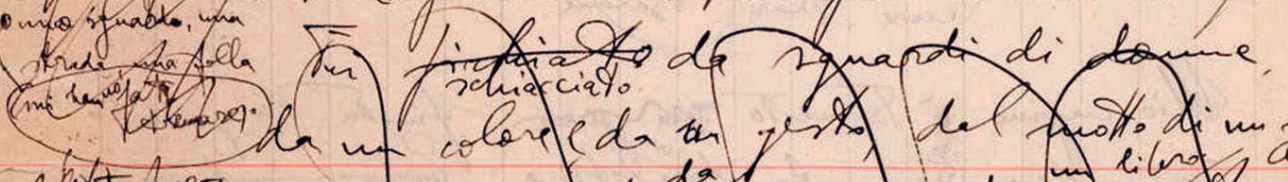

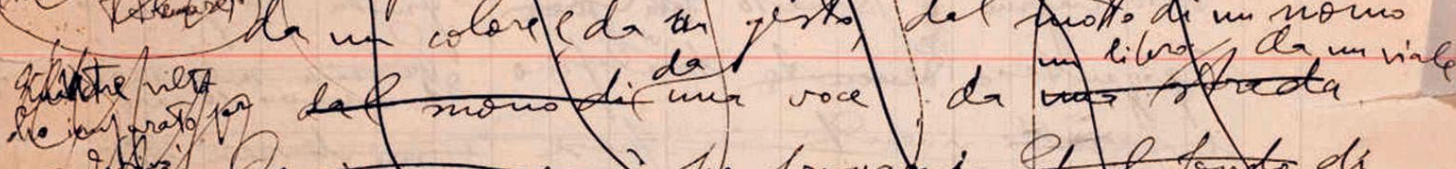

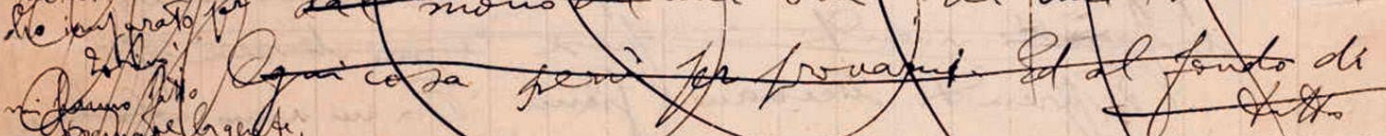
conteren

- Lasoen ralbas.

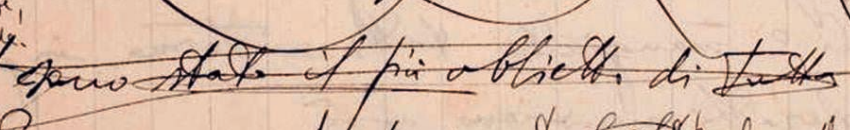

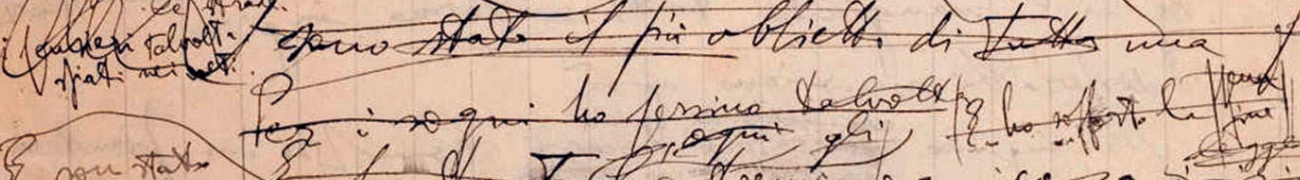

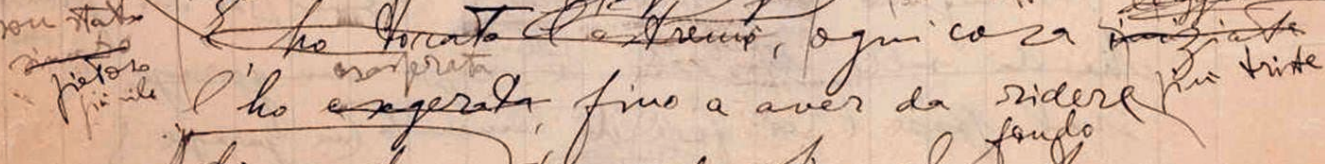
"di me ste no fimgendo, fino al finto

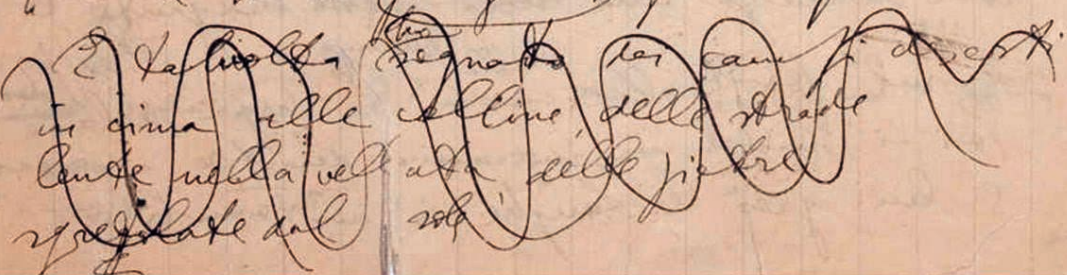

Fig. 4 : Feuillet AP III.1.2 r $^{\circ}$ (Guido Gozzano - Cesare Pavese, univ. di Torino, www.hyperpavese.it) 
Sur le feuillet suivant (AP III.1.2 $\mathrm{r}^{\circ 11}$, fig. 4), figure le développement de la deuxième partie de la digression, centrée sur l'adolescence. Dans un premier temps, elle se présente à l'opposé de la précédente, mais elle finit par être mise en place comme l'étape suivante d'un itinéraire; une expérience mûrie dans la ville aliénante, où le sentiment de solitude généré par la froideur et l'agressivité des rapports humains s'accompagne d'une forte frustration provoquée par les ambitions déçues et par les rêves échoués. Au départ petite parenthèse et simple instrument de dilatation temporelle, l'intermezzo ainsi amplifié assume de plus en plus les caractéristiques d'un approfondissement rétrospectif, qui dessine la lente construction de l'identité du personnage. Un flashback à la lumière duquel le retour du cousin, qui transmet une sécurité nouvelle, acquiert toute sa valeur (voir AP III.1.2 $\mathrm{v}^{\circ}:$ Ma $<$ ora $>$ tutto è lontano $<$ gioioso migliore finito $>$. La [illis. $]<$ forza $>$ pacata $<$ tranquilla $>/$ di moi del cugino moi umano compagno $>$ [cugino fraterno] vivo $<$ tornato mi [illis.] pulsa > è nel silenzio < moi sangue >).

Insérée quasiment d'un seul jet sous la poussée du souvenir des jeux d'enfants puis amplifiée, l'analyse en deux parties présentée dans l'intermezzo comportait un fort déséquilibre et un brusque changement de direction; Pavese sent donc la nécessité de revoir son plan originel pour restituer à la figure du cousin un poids capable de contrebalancer celui qu'il avait donné au protagoniste.

L'intermezzo trouve ainsi un complément avec la strophe qui raconte le retour, le nostos du cousin et ses différents choix sentimentaux et économiques (strophe 5 du poème publié); mais avant de l'écrire, Pavese trace des annotations qui figurent justement en AP III.1.2 $\mathrm{v}^{\circ}$ ( (Qualche volta gli scappa/parole/Pescare, Mari, Balene») (Quelquefois, des paroles/lui échappent/Pêcher, Mers, Baleines). Ces notes apparaissent comme un solide appui au moment où il a «perdu le fil», comme une méthode permettant de réordonner les priorités avant de s'apprêter à élaborer la suite du poème; nous allons le voir en analysant la dernière strophe.

\section{Langhe, baleines et Mers du Sud : une fin en expansion}

Après l'intermezzo, le premier brouillon développe (AP III.1.4 $\left.v^{\circ}\right)$ le cinquième point du plan $(\varepsilon)$, qui relié au point $\beta$ va donner naissance à la dernière strophe du poème publié. Pavese y associe le vent qui souffle sur la cime et celui qui «soffia nel grande deserto dell'Australia» (qui souffle dans le grand désert de l'Australie) (repris ensuite : «Io pensavo in quel mentre al sibilare / del vento sopra $i$ pascoli dell'Australia» (Moi je pensais pendant ce temps au sifflement / du vent sur les pâturages d'Australie), effacé, réécrit et enfin définitivement supprimé); mais le «vent» avec son «parfum» finit par contre par ouvrir la brève description du paysage nocturne ( Un profumo di terra e di vento ci avvolge [illis.] < leggero nel buio $>$ ) (Un parfum de terre et de vent nous enveloppe [illis.] $<$ léger dans l'obscurité >»), dont nous avons donc une première perception olfactive, liée à la «terre - - un élément fondamental du mythe pavésien - qui se substitue au «gravier» du plan $(\beta)$. L'odeur semble un instant remplir l'espace obscur («nel buio»), couvrant toutes les autres sensations, et c'est seulement dans un second temps que le poète insère une brève description visuelle ( Qualche lume lontano in distanza : cascine, automobili») (au loin, quelques lumières : des fermes, des autos) à laquelle s'ajoute une discrète perception auditive («che si sentono appena») (que l'on entend à peine). L'ouïe et la vue offrent des sensations qui contournent, voire détournent légèrement de la sensation plus profonde à laquelle on accède par l'odorat, comme si l'essence de la réalité gagnait à être perçue avec un sens plus subtil et moins rationnel. Et voilà que le rite s'accomplit et acquiert pour un instant une signification à travers «un profumo, un profumo di terra». C'est la terre qui donne la vie, mais qui en échange demande un sacrifice total. La nuit, avec ses lumières parcimonieuses et ses bruits étouffés, met en scène un événement presque métaphysique, un contact profond avec l'«être », réceptacle des forces ancestrales. Pavese développe ainsi le point qu'il indiquait entre parenthèses en $\varepsilon:$ : spirito» (esprit).

12. Voir par exemple la formule de AP III. $1.5 \mathrm{v}^{\circ}:$ : e dibattersi e scendere in mare < tendersi corde e lottare alla lancia > " modifiée en «e inseguirle e innalzarsi le code e lottare alla lancia», que l'on retrouve, après plusieurs autres modifications, en FE 5 II.38 00399200 W).

13. Ici, Pavese semble «exhiber» le souvenir du Moby Dick de Melville et de Song of Joys de Whitman. 
C'est seulement à la fin du poème qu'il récupère les trois motifs qui apparaissaient au centre du plan originel, et qui avaient été déplacés en dernière position en AP III.1.2 v ( Qualche volta gli scappa/parole/Pescare, Mari, Balene»). Ces trois motifs constituent le développement de la dernière partie du deuxième point $(\beta)$, du troisième $(\gamma)$ et du quatrième $(\delta)$, qui évoquent les maigres récits du cousin, les voyages en terre lointaine, les paysages exotiques et la fascination qu'ils suscitent chez le poète.

Ces points se concrétisent en AP III.1.5 $\mathrm{r}^{\circ}$ : le thème du paysage exotique est suivi de celui de la chasse à la baleine, la vraie passion du cousin, mais cette passion est introduite par son autre passion, celle des moteurs, qui est développée dans ce brouillon ( pensando < e pensa >ai suoi motori che ha imparatola conoscere a Frisco in California ») (pensant < et pense > à ses monteurs qu'il a appris à connaître à Frisco en Californie). Dans AP III.1.5

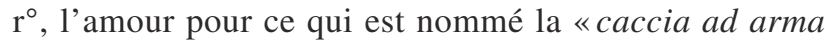
bianca» (chasse à l'arme blanche) est attesté par le fait qu'il en parle volontiers et qu'il en exhibe les souvenirs (une «fotografia» qu'il «mostra sognante» (montre en rêvant) et la «punta d'arpone» qui s'y substitue dans la seconde réélaboration, avant d'être éliminée elle aussi); la nostalgie pour une activité primitive compense l'attrait qu'il éprouve pour le monde de la mécanique et de la modernité ( «mio cugino, e rimpiange talvolta la sorte $<$ i progresso $><$ la sorte $>$ / che gli ha tolto di fare il fiociniere.» / «Pianterei tutto, moglie paese garage,/se si cacciasse ancora ad arma bianca») (mon cousin, et il regrette parfois le hasard le progrès le hasard / qui l'a empêché de travailler comme harponneur / «je planterais tout, femme village garage / si on chassait encore à l'arme blanche»). Mais ce motif lui aussi subit une réduction en volume, compensée par des précisions sur le rôle du cousin («da fuochista») (comme chauffeur) sur un bateau de pêche bien identifié ( «chiamato L'Arpone», puis remplacé par le «Cetaceo»).

La chasse à la baleine a subi plusieurs modifications, depuis le premier brouillon jusqu'au premier dactylogramme ${ }^{12}$, mais dans le premier plan un distique $(\gamma)$, y était déjà consacré 13 . Il devient un vers ( «Ha veduto fuggire balene tra schiume di sangue ») (Il a vu des baleines s'enfuir au milieu d'une écume de sang) qui non seulement restera quasi intact tout au long de la pénible élaboration du poème, mais semble l'orienter et la guider vers l'épilogue, scellant la figure du cousin, paradigme de l'expatrié, avec la richesse de ses signifiés épiques, mythiques, métaphysiques : l'aventure, le déracinement, la régression vers les origines, la lutte contre le Léviathan.

Dans la troisième et dernière reformulation de la fin, celle qui apparaît sur le premier brouillon, la «Bellezza» des «Mari del Sud» est placée après la mention des rêves du cousin, qui songe à repartir travailler comme harponneur. De ce fait, les indications portées sur les annotations de AP III.1.2 v («Pescare, Mari, Balene») sont inversées et réalisées selon un ordre différent («Pescare, Balene, $M a r i »)$, reportant à la clôture du poème le thème de l'attirance pour les îles tropicales.

À travers les rares informations données par le cousin, le protagoniste entrevoit la beauté des lieux exotiques. Dans les modifications apportées aux expressions (à la forme directe : «je lui dis que»), avec lesquelles le jeune garçon commente, on pourrait dire «interprète», l'expérience extraordinaire de l'adulte, on note un effort progressif du poète pour «représenter» la «Bellezza», en jouant sur quelques éléments du paysage (AP III.1.5 $\mathrm{r}^{\circ}$ et $\mathrm{v}^{\circ}$ ). Dans le premier jet ( Quando un giorno gli ho detto che ha visto levarsi il gran sole sulle isole più belle della terra») (Quand un jour je lui dis qu'il a vu se lever le grand soleil sur les îles les plus belles de la terre) ce sont les mots «il gran sole» et «le isole» qui sont mis en relief, et c'est plus marqué encore dans l'état suivant du vers ("gli ho detto ch'egli ha visto levarsi il sole enorme/sulle isole più belle della terra») (je lui ai dit qu'il a vu se lever le soleil énorme / sur les îles les plus belles de la terre), qui de plus actualise l'acte de parole, en éliminant l'indication «un jour» qui le renvoyait dans le passé par rapport au temps de la narration. La troisième et dernière réélaboration prolonge ce mouvement («Ma quando gli dico/ch'egli è tra i pochi <fortunati > che hanno visto l'alba $<$ l'aurora $>$ / sulle isole più belle della terra ») (mais lorsque je lui dis / qu'il est des rares $<$ heureux $>$ qui ont vu l'aube $<$ l'aurore $>$ / sur les plus belles îles de la terre). Elle marque le passage au présent mais substitue à l'allusion aux dimensions du soleil une indication sur les couleurs de l'aurore, et elle souligne la chance inouïe du cousin de pouvoir assister à tant de beauté («tra i pochi fortunati») (de ces heureux qui). 


\section{Le père aboli}

Le renversement le plus considérable du premier projet concerne la disparition de la figure du père du protagoniste, auquel pourtant deux points du plan étaient consacrés, qui plus est les deux derniers : $\zeta$ et $\Theta$. Il est donc étonnant qu'il n'en reste qu'une trace ténue dans le poème, une référence au père comme destinataire d'un billet envoyé par le cousin lointain (AP III.1.3 $\mathrm{r}^{\circ}$ : «Un inverno a mio padre, già morto, è arrivato < ò > un biglietto $<$ un cartoncino $>$ ») (Un hiver, pour mon père déjà mort est arrivé un billet $<$ une carte de vœux $>$ ).

Le père est donc l'intermédiaire pour arriver au cousin qui prend sa place, et qui d'ailleurs porte le même nom («arrivò un cartoncino firmato Pavese») (arriva une carte de vœux signée Pavese). On note l'indication explicite que le père était déjà décédé quand est arrivée la carte de vœux : l'insistance porte sur le rapport de parenté et sur la mort qui le brise en laissant le garçon orphelin, mais le billet lui permet de se sentir l'héritier de ce lien. Les «auguri di buona vendemmia» (vœux de bonne vendange) sur la carte postale sont importants car ils mettent en évidence aussi bien le lien à la terre que la valeur accordée au gain; et si le second élément peut paraître attendu, il acquiert davantage de relief quand il est mis en rapport avec les idées notées sur le plan à propos du père. De ces notations émerge en effet l'importance de deux traits distinctifs du personnage : l'amour pour la lecture et la volonté de «far soldi in famiglia» (gagner de l'argent en famille). Pour le cousin également, l'argent est la seule priorité, même s'il est en quête d'une affirmation personnelle.

Sur le plan, la figure paternelle est entourée d'un halo de pessimisme et de défaite, symboles de la dureté de la vie; dans le poème, il en reste seulement une allusion fugace, indissolublement liée à la mort. Elle prend la place d'une référence à la guerre (Un inverno era < un pò > prima della guerra $\rightarrow$ Un inverno a mio padre, già morto, arrivò un cartoncino) (un hiver, c'était $<$ un peu $>$ avant la guerre $\rightarrow$ Un hiver, pour mon père déjà mort arriva une carte de vœux), mais ce n'est pas une simple indication temporelle, plutôt un enchevêtrement complexe de renvois symboliques, qui instaure un pont entre les deux protagonistes et qui donne à l'arrivée du billet la valeur d'un événement salvateur. La carte de vœux semble outrepasser la mort du destinataire et en même temps transmettre à l'orphelin sécurité et rêves. Le soulignement, même implicite, de cette valeur du billet révèle, presque à la manière d'une synecdoque, une caractéristique fondamentale du cousin, qui l'oppose nettement au père ; il a survécu aux longs voyages, aux travaux les plus durs, aux climats les plus divers, et enfin à la guerre, quand tous au village le donnaient pour mort après seulement quelques années d'absence. Il revient vivant, enrichi qui plus est. Non seulement il a su s'adapter et se bâtir une vie satisfaisante, mais il a vaincu la mort. Tout dans son profil, sa silhouette de géant, ses vêtements blancs et ses projets farfelus, met en évidence son caractère surhumain. Sa force lui vient d'avoir accepté de se mesurer à l'altérité, d'avoir expérimenté sur lui-même des conditions extrêmes et d'avoir connu dans le monde la souffrance, la faim et la mort (AP III.1.4 $\mathrm{v}^{\circ}$ : «Mio cugino ha sofferto la fame nel mondo e la morte»). Une dimension héroïque qui rachète la faiblesse congénitale de sa lignée, quasi destinée à une mort précoce, et en exalte les caractéristiques exceptionnelles (voir la chute de la strophe 1). Ainsi, il faut retourner à l'avant-texte pour bien mesurer l'importance donnée par Pavese au thème de l'effort et de la souffrance dans sa construction du personnage du cousin; dans le texte publié, il n'en reste que quelques traces ou allusions.

\section{Pour conclure}

À partir du plan de «Les mers du sud», dont l'importance peut être saisie grâce à la méthodologie génétique, les ajouts, suppressions et permutations documentent la genèse du poème dans une tension vers une mythographie personnelle

14. Voir la note «col cugino», point $\beta$ du plan. Le cousin n'était nommé que deux fois par son nom en AP III.1.3 $\mathrm{v}^{\circ}$ : «sai che è tornato Silvio» (Tu sais que Silvio est revenu).

15. Marziano Guglielminetti, « Racconto e canto nella metrica di Pavese », Sigma, $n^{\circ} 1,3-4,1964$, p. 22-33; p. 27.

16. On pourrait ainsi étudier, à partir de «in silenzio», point $\alpha$ du plan, le développement de ce thème du silence dans le poème : dans la première strophe Pavese aboutit, après quelques hésitations, à une structure croisée générée par les répétitions et la derivatio (silence, taciturne, se taire, silence), présentant le silence comme un trait des deux personnages; dans la seule autre occurrence, à la fin du poème (v. 89), le silence sera la métaphore de la mort («il silenzio che dura»). 
qui a déjà des ambitions plus vastes. L'analyse de détail du poème nous a confirmé que ce processus prenait deux directions de correction dominantes et systématiques dans l'écriture de Pavese.

Premièrement, le poète renonce à des thèmes pour lesquels il n'a pas trouvé de place ou qu'il juge désormais non essentiels; ainsi non seulement il élimine un surplus d'informations mais travaille également sur son propre langage par soustraction, à la recherche d'une condensation sémantique en images. Cette technique triomphe par exemple dans le grand final du poème, dans le «jour déjà vieux de l'aurore », où ressortent avec force les motifs supprimés de la fatigue et de la faim.

Mais par ailleurs, Pavese construit laborieusement une trame serrée de répétitions, proches ou lointaines, dont on mesure la portée en rapport avec celles qui à l'inverse ont été supprimées : c'est très clair dans l'insistance sur le syntagme «mio cugino 14 », dont restent seulement les occurrences possédant une fonction anaphorique (v. 3, 9, 54, 59, 80, 90), particulièrement recherchée par l'auteur. L'intention n'est pas seulement rythmique - donner au poème une cadence épique - ; ces répétitions servent aussi à mettre en évidence un thème qui devient le pivot du récit, à créer une image-mythe qui sera complétée par ajouts successifs et donc à tisser une toile de mots-clés qui, pour le dire avec Guglielminetti, deviennent des «points d'orientation lumineux 15 », capables de relier entre elles les différentes images 16. C'est également à travers l'usage des répétitions que Pavese construit sa mythographie personnelle (la communion dans le silence avec le cousin, irrité par la communauté ; la stature héroïque de l'émigrant, incarnation du rêve d'aventure dans les mers féroces et peuplées de baleines en fuite), qui a déjà le parfum d'une mythographie collective (le mythe de l'enfance perdue, de l'identité, des racines, du sang).

Ainsi, la reconstitution de la genèse d'un des poèmes les plus significatifs de l'auteur permet de saisir sa poétique en acte. Avec «Les mers du sud», Pavese «découvre»sa propre cadence poétique et «invente» ce style balançant entre récit épico-mythique et concentration poétique, qui fera de Lavorare stanca - son unique recueil publié - un ouvrage qui est à la fois un exorde et une somme poétique.

Traduit de l'italien par Claire Riffard

\section{MARI DEL SUD 17}

[1] Camminiamo una sera sul fianco di un colle, in silenzio. Nell'ombra del tardo crepuscolo mio cugino è un gigante vestito di bianco, che si muove pacato, abbronzato nel volto, taciturno. Tacere è la nostra virtù.

Qualche nostro antenato dev'essere stato ben solo - un grand'uomo tra idioti o un povero folle per insegnare ai suoi tanto silenzio.

[2] Mio cugino ha parlato stasera. Mi ha chiesto se salivo con lui : dalla vetta si scorge nelle notti serene il riflesso del faro lontano, di Torino. "Tu che abiti a Torino..." mi ha detto "... ma hai ragione. La vita va vissuta lontano dal paese : si profitta e si gode, e poi, quando si torna, come me, a quarant'anni, si trova tutto nuovo. Le Langhe non si perdono". Tutto questo mi ha detto e non parla italiano, ma adopera lento il dialetto, che, come le pietre di questo stesso colle, è scabro tanto che vent'anni di idiomi e di oceani diversi non gliel'hanno scalfito. E cammina per l'erta con lo sguardo raccolto che ho visto, bambino, usare ai contadini un poco stanchi.

[3] Vent'anni è stato in giro per il mondo. Se n'andò ch'io ero ancora un bambino portato da donne e lo dissero morto. Sentii poi parlarne da donne, come in favola, talvolta; ma gli uomini, più gravi, lo scordarono. Un inverno a mio padre già morto arrivò un cartoncino con un gran francobollo verdastro di navi in un porto e augurî di buona vendemmia. Fu un grande stupore, ma il bambino cresciuto spiegò avidamente che il biglietto veniva da un'isola detta Tasmania circondata da un mare più azzurro, feroce di squali, nel Pacifico, a sud dell'Australia. E aggiunse che certo il cugino pescava le perle. E staccò il francobollo. Tutti diedero un loro parere, ma tutti conclusero che, se non era morto, morirebbe.

Poi scordarono tutti e passò molto tempo.

[4] Oh da quando ho giocato ai pirati malesi, quanto tempo è trascorso. E dall'ultima volta che son sceso a bagnarmi in un punto mortale

17. Première édition revue par l'auteur (Solaria, 1936) 
e ho inseguito un compagno di giochi su un albero spaccandone i bei rami e ho rotto la testa a un rivale e sono stato picchiato, quanta vita è trascorsa. Altri giorni, altri giochi, altri squassi del sangue dinanzi a rivali più elusivi : i pensieri ed $i$ sogni.

La città mi ha insegnato infinite paure : una folla, una strada mi han fatto tremare, un pensiero talvolta, spiato su un viso. Sento ancora negli occhi la luce beffarda dei lampioni a migliaia sul gran scalpiccìo.

[5] Mio cugino è tornato, finita la guerra, gigantesco, fra i pochi. E aveva denaro. I parenti dicevano piano : "Fra un anno, a dir molto, se li è mangiati tutti e torna in giro.

I disperati muoiono così".

Mio cugino ha una faccia recisa. Comprò un pianterreno nel paese e ci fece riuscire un garage di cemento con dinanzi fiammante la pila per dar la benzina e sul ponte ben grossa alla curva una targa-réclame. Poi ci mise un meccanico dentro a ricevere i soldi e lui girò tutte le Langhe fumando.

S'era intanto sposato, in paese. Pigliò una ragazza esile e bionda come le straniere che aveva certo un giorno incontrato nel mondo. Ma uscì ancora da solo. Vestito di bianco, con le mani alla schiena e il volto abbronzato, al mattino batteva le fiere e con aria sorniona contrattava i cavalli. Spiegò poi a me, quando fallì il disegno, che il suo piano era stato di togliere tutte le bestie alla valle e obbligare la gente a comprargli i motori.
"Ma la bestia" diceva "più grossa di tutte, sono stato io a pensarlo. Dovevo sapere che qui buoi e persone son tutta una razza".

[6] Camminiamo da più di mezz'ora. La vetta è vicina, sempre aumenta d'intorno il frusciare e il fischiare del vento. Mio cugino si ferma d'un tratto e si volge : "Quest'anno scrivo sul manifesto : - Santo Stefano è sempre stato il primo nelle feste della valle del Belbo - e che la dicano quei di Canelli". Poi riprende l'erta. Un profumo di terra e di vento ci avvolge nel buio, qualche lume in distanza : cascine, automobili che si sentono appena; e io penso alla forza che mi ha reso quest'uomo, strappandolo al mare, alle terre lontane, al silenzio che dura.

Mio cugino non parla dei viaggi compiuti.

Dice asciutto che è stato in quel luogo e in quell'altro e pensa ai suoi motori.

Solo un sogno

gli è rimasto nel sangue : ha incrociato una volta, da fuochista su un legno olandese da pesca, il Cetaceo, e ha veduto volare i ramponi pesanti nel sole, ha veduto fuggire balene tra schiume di sangue e inseguirle e innalzarsi le code e lottare alla lancia. Me ne accenna talvolta.

Ma quando gli dico ch'egli è tra i fortunati che han visto l'aurora sulle isole più belle della terra, al ricordo sorride e risponde che il sole si levava che il giorno era vecchio per loro.

Lavorare stanca (Solaria, 1936) 
Maura Bonfiglio a obtenu son diplôme de Master 2 à Cagliari (Italie), en juillet 2010, sur le thème : « Dino Campana, Canti Orfici, fra tradizione e innovazione». Elle a soutenu en 2016 à Cagliari une thèse doctorale, «Le carte del primo Lavorare stanca» - une étude philologique et génétique des manuscrits du premier recueil poétique de Cesare Pavese. Ses recherches portent sur la littérature italienne contemporaine, la philologie d'auteur et la critique génétique.

bonfiglio.maura@gmail.com

Dans cet article sont analysées les étapes les plus significatives de l'élaboration de «I mari del sud», le poème qui ouvre Lavorare stanca, seul recueil de poésies publié de son vivant par Cesare Pavese. Le projet initial, un plan thématique organisé en sections, subit rapidement une modification importante de l'ordre et des thèmes choisis, conduisant Pavese à compléter ce premier plan par un second. La difficile mise en place thématique du poème, à grand renfort de déplacements, d'ajouts, de changements et de suppressions, s'accompagne d'une recherche expressive qui creuse progressivement le langage, vise la valorisation du détail et du concret, pour aboutir à une condensation en images. Une recherche qui toutefois ne craint pas les répétitions, mais les utilise pour cadencer le discours et lui donner une épaisseur épique, en mettant en évidence certains thèmes clés.

In this article we analyze the most significant stages of the elaboration of "I mari del Sud", the first poem in Lavorare stanca, the only volume of poetry published by Cesare Pavese in his lifetime. The initial project, a thematic outline organized in sections, was soon greatly modified in its structure and selected themes, leading Pavese to complete this first outline with a second one. The difficult thematic arrangement of the poem, through many displacements, additions, changes and suppressions, with a search for expressivity deepening the language, aiming at the enhancement of the detail and the concrete, finally leads to a condensation of images. A search however that does not shy from repetitions but uses them to rhythm speech and give it an epic density, by highlighting certain key themes.

Im vorliegenden Artikel analysieren wir die wichtigsten Etappen der Ausarbeitung von „I mari del sud“, des Eröffnungsgedichts von Lavorare stanca, der einzigen Gedichtsammlung, die Cesare Pavese selbst zu Lebzeiten veröffentlichte. Das ursprüngliche Projekt, ein Plan für eine thematische Anordnung in einzelnen Abschnitten, erfährt bald eine bedeutende Modifikation hinsichtlich der Reihenfolge und der ausgewählten Themen. Pavese lässt so dem ersten einen zweiten Plan folgen. Er arbeitet lange und mühevoll an der thematischen Strukturierung des Gedichts, macht Verschiebungen, Zusätze, Veränderungen, nimmt Streichungen vor. Parallel dazu arbeitet er am sprachlichen Ausdruck, geht nach und nach der Sprache auf den Grund, nimmt eine Neubewertung des Details und des Konkreten vor, um eine Verdichtung in Bildern herbeizuführen. Eine Suche, die vor Wiederholungen nicht zurück schreckt sie jedoch verwendet, um den Diskurs rhythmisch zu strukturieren und ihm epische Breite zu geben, sodass gewisse Schlüsselthemen hervortreten.

\section{Coups de sonde génétiques dans "Les mers du sud"}

En este artículo se analizan las etapas más significativas de la elaboración de "I mari del sud", poema que abre Lavorare stanca, único libro de poesía publicado en vida por Cesare Pavese. El proyecto inicial -un plan temático organizado en secciones-sufre rápidamente una modificación importante en su ordenamiento y en los temas escogidos, lo que lleva a Pavese a completar este primer plan con un segundo. La difícil elaboración temática del poema -a través de múltiples desplazamientos, añadidos, cambios y supresiones- va acompañada por una búsqueda expresiva, que horada progresivamente el lenguaje, buscando una valorización del detalle y de lo concreto, hasta lograr una condensación en imágenes. Una búsqueda que, no obstante, no teme las repeticiones, sino que las utiliza para acompasar el discurso, otorgándole un espesor épico y poniendo en evidencia algunos temas clave.

Neste artigo são analisadas as mais importantes etapas da elaboração de "I mari del sud", o poema de abertura de Lavorare stanca, única colectânea de poemas publicada em vida por Cesare Pavese. O projeto inicial, organizado em secções temáticas, rapidamente foi alterado na ordem e nos temas escolhidos. A inserção temática do poema foi difícil, exigindo muitas alterações de escrita, com uma pesquisa de linguagem progressivamente mais expressiva, valorização do pormenor concreto, imagens condensadas. As repetições abundam como forma de cadenciar o discurso e lhe dar espessura épica, evidenciada em certos temas chave.

Sono prese in esame le tappe più significative dell'elaborazione de "I mari del Sud", la lirica che apre Lavorare stanca, l'unica vera raccolta poetica pubblicata da Cesare Pavese. Un primo progetto, testimoniato da una traccia tematica per punti, subisce ben presto una ristrutturazione che comporta un profondo ripensamento dell'ordine e della selezione dei temi da sviluppare e spiega l'esigenza di Pavese di integrare il primo schema con un secondo. Di pari passo con la faticosa realizzazione di una struttura tematica, costruita a forza di spostamenti, aggiunte, ripensamenti, e espunzioni, si svolge una ricerca espressiva che progressivamente scava il linguaggio mirando alla valorizzazione del dettaglio, alla ricerca di concretezza e aderenza alle cose che a sua volta sfocia nella condensazione in immagini. Una ricerca che, però, non teme le ripetizioni, utilizzandole in maniera calcolata per cadenzare il discorso e donargli spessore epico, mettendo in evidenza alcune tematiche chiave. 